The following article was initially presented during the M\&M2001 Core Facility Management session organized by Debby Sherman, Purdue University. The original recorded version was lost due to a technical problem and Michael Postek very generously reconstructed his portion of the presentation. Questions posed to Michael by those attending (indicated by bullet) and his responses are at the end of his presentation. This is a companion presentation to that of Dr. John McCaffrey on TEM calibration (Microscopy Today Jan/Feb 2002).

\section{Calibration of Electron Microscopes: How to do this, how often, pit-falls and problems}

\author{
Michael T. Postek \\ National Institute of Standards and Technology' \\ postek@nist.gov
}

SEM Instrument calibration is something most people don't always realize they need to do. Images look about right so the magnification seems close enough. Today, measurements are being done in specialized scanned beam metrology instruments everyday, so correct magnification calibration is very important. Calibration is probably more acknowledged from the point of the transmission electron microscope than for the scanning microscope. But, it still needs to be done. Today, people can buy SEM's that cost around $\$ 100,000$ (refurbished ones somewhat less) to $\$ 2.5 \mathrm{M}$ with one instrument model from one manufacturer costing about $\$ 8 \mathrm{M}$. Believe it or not, often the instrument buyer thinks that as soon as they get the instrument into their facility (since it is new and they paid a lot of money for it) that it is perfect and everything is correct. Often that is not the case - as you will soon see. Proper and accurate instrument calibration at the factory is the first issue and second, things can change during shipment. So, you must keep in mind that it is your responsibility as the user to check that the magnification calibration is correct, as it is primary to the proper operation of the SEM.

Due to tight time constraints, we are only going to deal with the calibration of the " $X$ " and " $Y$ " scans today. As SEM owners or operators it is something we need to check and re-check on a periodic basis. You have to be aware that the electronics can drift and so the instrument needs to be re-calibrated periodically. Standards of some sort are needed. I will mention a few of the calibration standards during this presentation.

The SEM has inherent systematic problems that should be understood-especially if one wants to do accurate measurements. In order to develop accurate standards for the SEM, we at NIST have studied and published our findings over the years on this topic. ${ }^{2}$

Again, because of time limitations, this talk will only be restricted to instrument calibration and not other instrument or operator errors that can also present problems. When it comes to the instrument calibration itself, each SEM has its own unique design and calibration procedure. I recommend that you or your technologist spend time with your service engineer, while at your site, in order to find out the calibration procedure particular to the instrument that you have in your laboratory. You should come to an understanding with the service engineer regarding frequency of calibration testing and whose responsibility it is to make the check and determination of when a recalibration procedure is necessary. With many of the newer instruments, re-calibration is adjusted in software or it may actually be a hardware adjustment requiring tweaking the instrument itself - all depends upon the design. But, rest assured, that whoever does the calibration, you are the one responsible for providing the appropriate SEM magnification calibration standard. If you require traceability to National or International standards of length, as many people do in industry today, you need to provide the traceable standard. Don't expect the service engineer to have one. But, if you just give the field service engineer the opportunity to do the calibration without any direction, most likely the engineer will just pull out and use a standard transmission electron microscope grid. I am sure each of you know what a $3 \mathrm{~mm}$, copper, TEM grid is and looks like. This grid is supposed to be round and supposed to be $3 \mathrm{~mm}$, but there is no standard related to it other than the fact that it fits within the holder of the appropriate TEM. All a $3 \mathrm{~mm}$ grid can tell you, or the engineer, is that the scans are orthogonal to each other and the same size. So round things will appear to be round and square things will be square. But, this procedure says nothing about the proper magnification associated with the instrument.

The traditional Standard Reference Material (SRM) for magnification calibration and traceability is NBS/NIST SRM 484. It is a sample that has accurate pitch dimensions for SEM magnification calibration. What I want to do first is to give you an idea of how well SEMs are calibrated today. These data are a little bit on the old side but, I believe that even if I redid this work today, it would probably come out about the same. I organized a SEM calibration interlaboratory study in 1993. We surveyed about 60 instruments across the U.S. from; universities, commercial laboratories, and industry. We studied the magnification calibration itself, the adjustment of the $x-y$ calibration scans for squareness, photographic CRT adjustment, accelerating voltage compensation and dimensional measurements. This work was presented at MSA $^{3}$ published in other journals ${ }^{4}$ and in the National Bureau of Standards (NIST) Journal of Research. ${ }^{5}$

How Well Are SEMs Calibrated Today?

A prototype SEM magnification standard referred to as Reference Material (RM 8090) was circulated to about 60 laboratories in an interlaboratory study. The participants were requested to provide micrographs of specific sections of the SRM prototype at eight specific magnification points. These points were specifically chosen to test the magnification range calibration of the instrument under test. Figure 1 shows the magnification calibration performance of a new instrument essentially "right out of the box" and the data from a second instrument of the same manufacturer and model from a second laboratory. Note that the new instrument was about 8-10\% mis-calibrated (magnification too large) in its magnification calibration performance. That 8-10\% might be within the manufacturers specification. But, is it within yours? The second instrument from a different laboratory demonstrated that the owner of that instrument understood the need for magnification calibration and had accurately calibrated the instrument throughout the magnification ranges tested. It also demonstrates that, given the proper attention, very accurate magnification calibration is possible.

Sometimes, we work in a facility that has several instruments available for our use. One day the investigator might use one instrument and on another day a different one depending upon the sign-up schedule employed. In order to ensure data continuity between instruments, clearly, magnification matching across these instruments is needed. Figure 2a shows two instruments from the same laboratory operating essentially side-by-side in different rooms. Note that Instrument 1 has as much as $20 \%$ magnification 


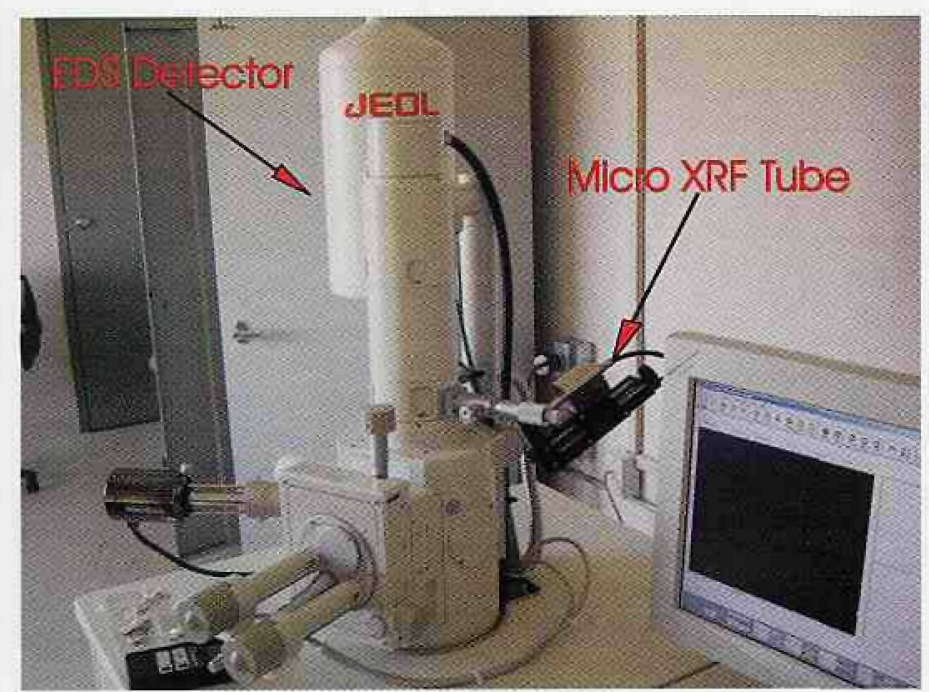

Tace analysis.

\section{race Analysis In Your SEM!}

It's now possible to do trace analysis using your existing $S E M$. IXRF Systems introduces their exclusive new option for tr

\section{Taking SEM-EDS Integration To The Next Level!}

Add the new $f X$ option to your existing SEM-EDS system and get the benefit of trace analysis, on any sample. The sample doesn't even need to be coated or analyzed in a vacuum! The new $f X$ option not only allows trace analysis down to a few ppm, but can also analyze major elements to complement EDS Microanalysis.

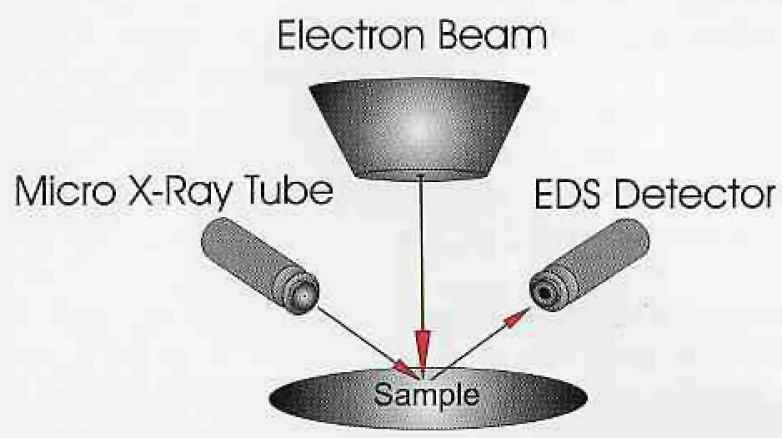

\section{How Does It Work?}

The new $f X$ option, from IXRF, consists of a miniature $\mathrm{X}$-ray tube that is closely coupled with both the sample and EDS detector, within the tight constraints of the SEM chamber.

The $f X$ tube is mounted on a slide so it can be positioned close to the sample for small-area analysis $(<1 \mathrm{~mm})$ or further away for larger-area (up to at least $10 \mathrm{~mm}$ ) analysis.
Lower Backgrounds

by XRF can give

detection limits in

the low ppm range.
Analyze bulk or thin-film samples for both thickness and composition.

\section{Expanding Your Capability!}

Using the technique of ED-XRF (Energy

Dispersive X-ray Fluorescence) you get its proven advantages of sensitivity and low backgrounds. These benefits mean that low concentrations, which cannot be detected using conventional electron-beam excitation, can easily be analyzed using IXRF's $f X$ option.

Peak-to-background ratios using XRF are typically up to 10 to 100 times higher than SEMEDS values. For the first time the analyst can get complete SEM-EDS and XRF in one system.

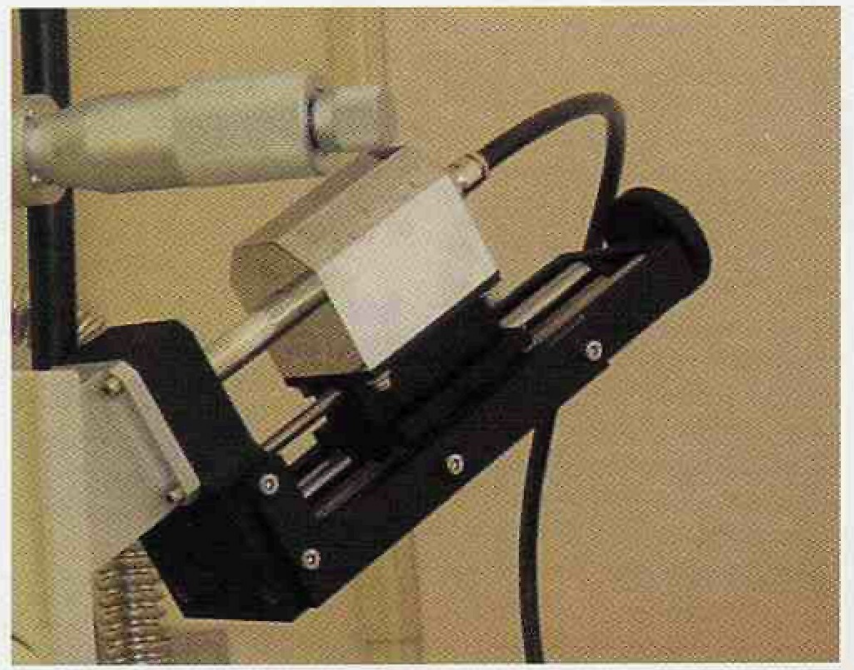

\section{Adaptation!}

Just about every SEM can be adapted. IXRF provides complete installation of the $f X$ option. The only thing needed is an available port.

Call or email us for your specific configuration.

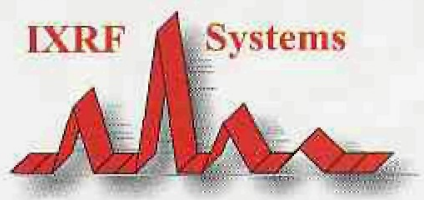

IXRF Systems, Inc. 15715 Brookford Dr. Houston, Texas, 77059 Tel:281/286-6485 Fax:281/286-2660 www.ixrfsystems.com 


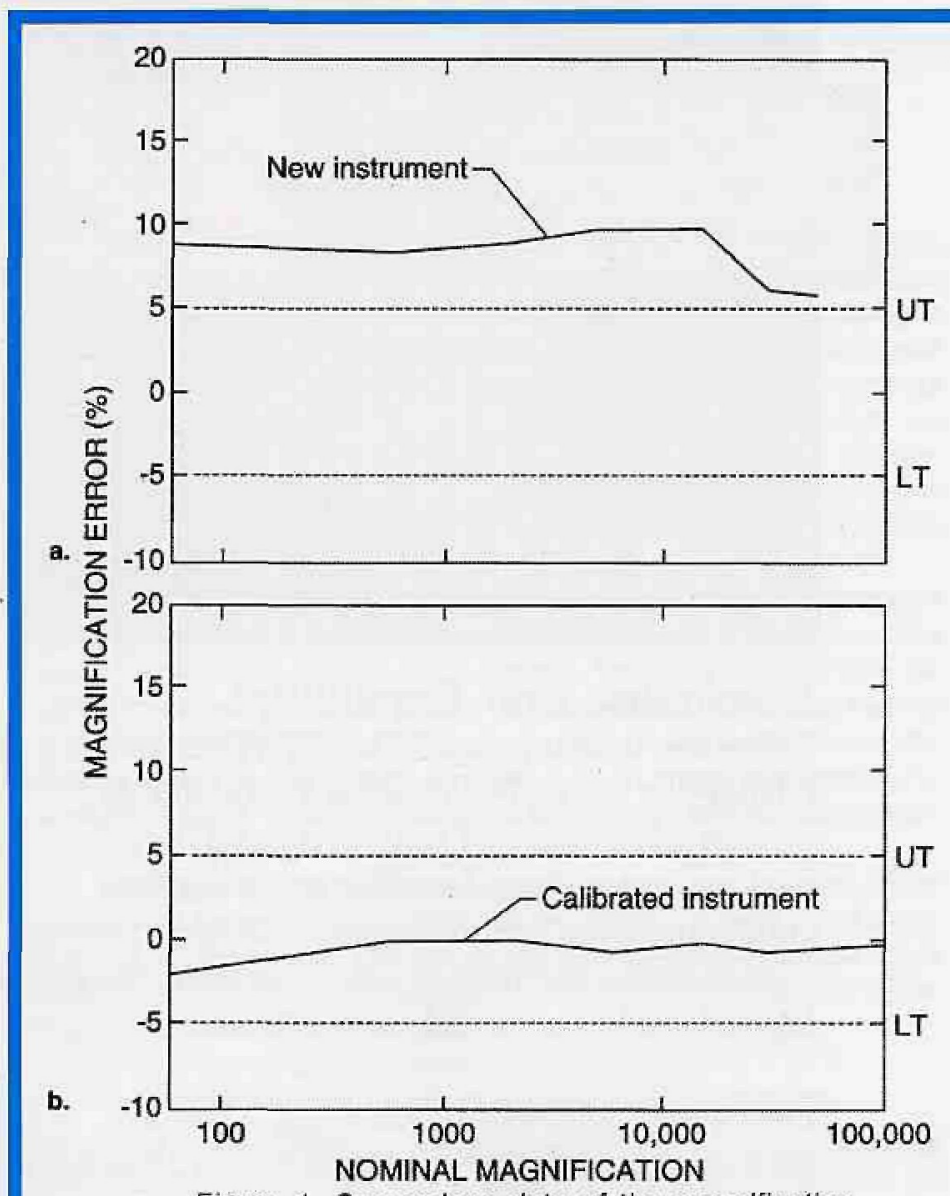

Figure 1. Comparison data of the magnification calibration of two instruments of similar manufacture and model, but from different laboratories, a) Data from a new instrument and b) Data from a properly calibrated instrument.

mis-calibration (too large) as compared to Instrument 2. This means that if an investigator used Instrument 1 one day and Instrument 2 another day on similar samples, a new species (if size is a consideration) could be discovered. Figure $2 \mathrm{~b}$ shows the calibration data for four instruments from a single laboratory facility that have all been matched to each other.

I refer you to the papers regarding the full study, but to make a long story short, the standard deviation of all the instruments was about $12 \%$ with a maximum error of as much as $63 \%$.

\section{Calibration Standards}

One of NIST's roles is the development of standards traceable to the National Standard of Length - in this case, the meter. If one wants to develop a calibration standard for a scanning electron microscope; one must optimize the user base for such a standard.

Thus, there are a number of considerations that have been taken into account when a standard is defined. ${ }^{6}$ First, the standard needs to be relevant to the needs of the majority of the users. Today, SEM instrumentation has a wide range of capabilities and a standard should be capable of being used with almost any of these instruments. Modern instruments function over a wide range of performance parameters. One main parameter is accelerating voltage. So a standard needs to be able to be used over the range of accelerating voltages available and being used on that instrument. Standards are expensive so it should be resilient and cleanable. The overall thickness of the standard should be that of a silicon wafer so that it can be used in wafer inspection instruments or placed on a stub for more conventional laboratory instruments. Since physical rotation of a sample is not possible in

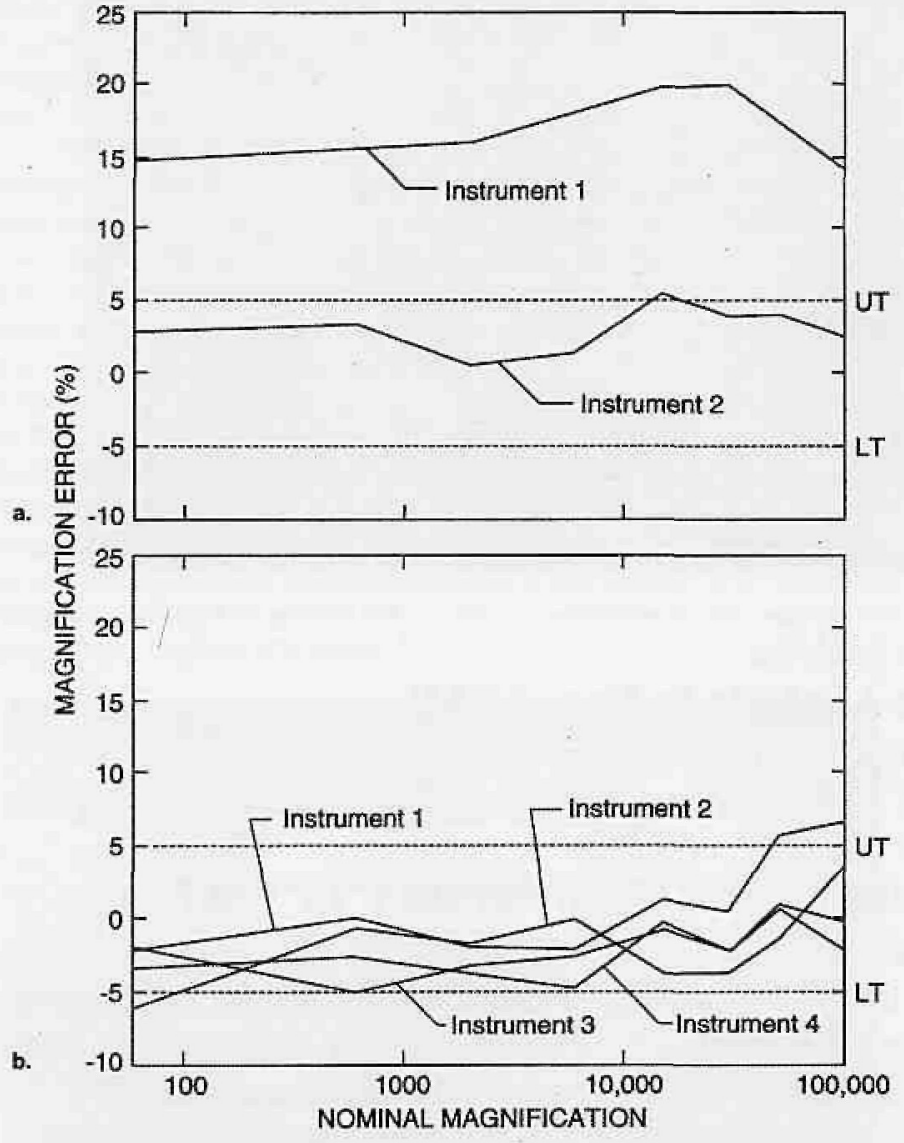

Figure 2. Data demonstrating the calibration of multiple instruments from a single laboratory. a) Two instruments from the same laboratory demonstrating a $15-20 \%$ magnification discrepancy between instruments and b) Comparison of four instruments within a single lab.

many of the newer semiconductor production instruments, calibration structures in both $X$ and in $Y$ are needed. Modern instrumentation has a wide variety of different lens designs so a calibration standard must be non-magnetic and finally, the measurement of the sample needs only be based on a pitch (displacement) between lines and should not be based on a width of a line, ${ }^{7}$

Calibration standards are available from a number of sources. Standards are available from instrument manufacturers, commercial suppliers, in-house standards laboratories and National Measurement Institutes such as NIST. In this presentation, I will only address the standards available or soon to be available from NIST. More information about these standards can be obtained by contacting the NIST office of Standard Reference Materials or accessing the on-line catalog (http://ts.nist.gov/ts/ htdocs/230/232/232.htm).

SRM 484. Standard Reference Material (SRM) 484 is the original SEM magnification standard initially issued by the National Bureau of Standards and now NIST. It is composed of electro-deposited parallel gold lines on a nickel substrate. The substrate is placed within a metallurgical mount so that the layers are viewed in crosssection. The surface is highly polished. The atomic number contrast between the gold and the nickel is sufficient for imaging and accurate instrument calibration to the measured and certified quantity. SRM 484 is accurately calibrated using laser interferometry in a specially designed metrology microscope at NIST. This standard was designed before the emphasis on low 
accelerating voltage SEM operation and thus is more suitable to high accelerating voltage applications.

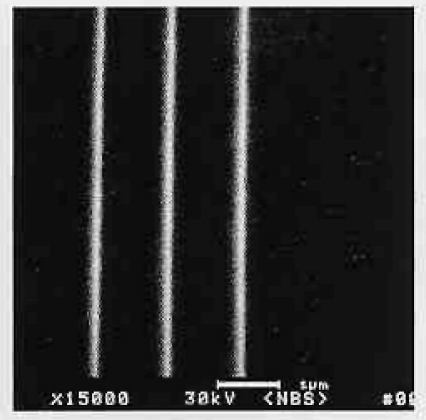

a

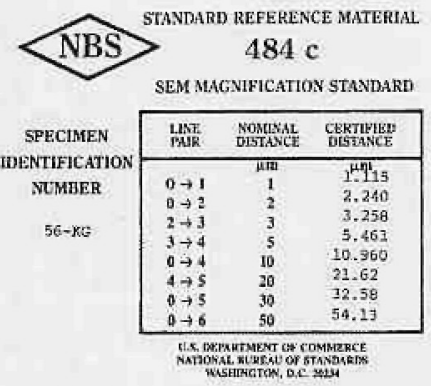

b.
Figure 3. SEM calibration with SRM 484. a) SEM image of the $0,1,2$, and 3 gold lines and $b)$ SRM documentation showing of an older version of SRM 484 the certified distances measured using a specialized instrument at NBS/NIST in order to provide traceability.

SRM 2090/RM8090. Prototype samples of the Standard Reference Material 2090 were used in the interlaboratory study referenced above. The first production version of that standard was released to the industry as Reference Material 8090 . RM 8090 was intended to be a pre-release for the SRM. RM 8090 and SRM 2090 are intended primarily for use in calibrating the magnification scale of a scanning electron microscope (SEM) over a wide range of magnifications, from less than $100 X$ to greater than $300000 X$. RM 8090 contains structures in both $X$ and $Y$ dimensions, ranging in nominal pitch from $0.2 \mu$

useful at both high and low accelerating voltages. RM 8090 is the thickness of a silicon wafer and thus, can be inserted in the modern automated measurement systems or the artifact can be mounted directly to a specimen stub. Most laboratory SEMs and many of the dedicated in-line metrology SEM-based instruments require a number of calibration structures to cover the full range of magnification. This standard is specifically designed to meet that need as shown in Figure 4. RM 8090 is an advance issue of SRM 2090 intended to satisfy industry need during the final phases of the SRM 2090 development cycle. SRM 2090 will be fully characterized and certified according to NIST procedure.
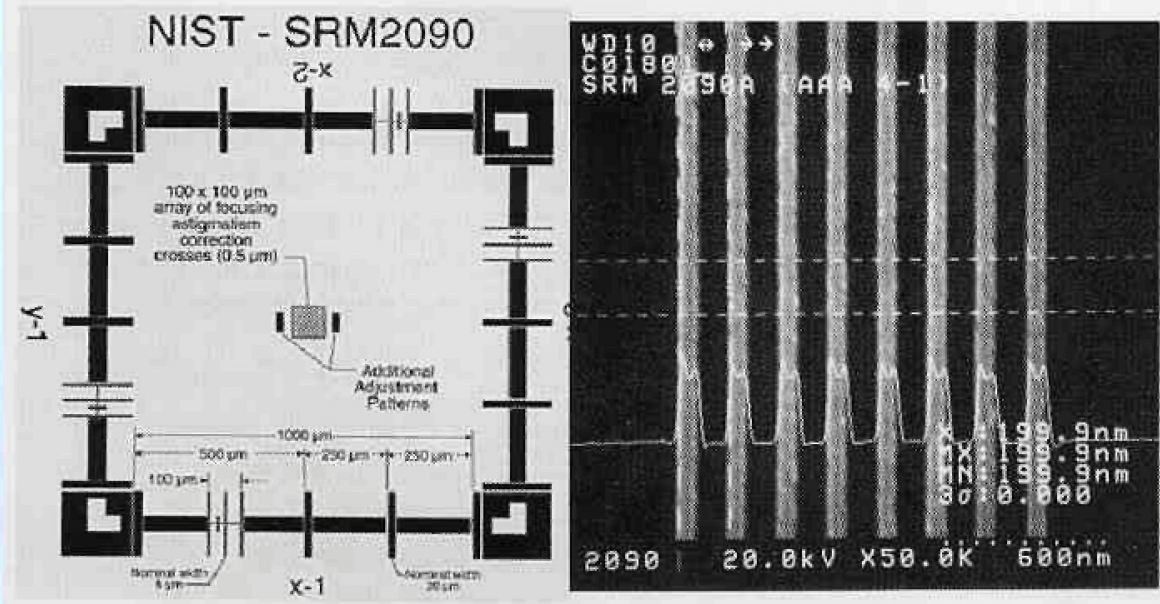

Figure 4. SRM 2090/RM 8090 scanning electron microscope calibration standard. a) Low magnification drawing with several of the nominal pitched listed and b) SEM Micrograph of SRM 2090 showing the fine line structure at $50,000 \times$ magnification.
Problems associated with the direct-write e-beam fabrication of the SRM have delayed its full release. Currently, samples are being fabricated and release is expected by the Fall 2002.

Take-home Lesson

The final take-home lesson from this short lecture and introduction into the faults and foibles of the scanning electron microscope is that it is the user's responsibility to make sure that the instrument is properly calibrated. Furthermore, it is the users responsibility to check the instrument on a periodic basis to insure that calibration drift has not occurred.

\section{Questions from the Audience}

Q: I ran into some problems in calibration in that I want an inexpensive calibration sample that I can hand to a student. I want to have it around so users can routinely throw it into the microscope and take calibration images at the magnifications that they are using at that particular time. In SEM we can use the replica lattice gratings that are readily available through the EM supply houses for magnifications of about $1000 x$ and up to about $50,000 \mathrm{x}$ but we have a lot of people doing things below $1000 \mathrm{x}$. And they need to have some sort of calibration as well. So it is a problem of what we should use at the very low magnifications in an SEM. Again, it needs to be very affordable so that students can routinely use it. We would appreciate knowing about any suggestions you might have for those very practical types of samples.

M. Postek: In answer to your question, I have already solved that problem for you with the 2090 sample, because it goes from $3 \mathrm{~mm}$ all the way down to $0.2 \mu \mathrm{m}$ pitch. But what you can do in regards to the expense issue, would be to develop a secondary standard based on that. If you have a grating or something else in your facility that you feel confident with that meets the criteria of resilience, if nothing else, you can use the traceable standard to provide a trace-ability chain to that standard. You might then distribute that relatively cheap standard to the students so they can check the magnification. I don't have anything in mind at this moment that would help you in that area but there might be something. But you can develop your own trace-ability chain in that respect and that is perfectly correct in doing that.

$\mathrm{Q}$ : Obviously, if you are working with something that is crucial for measurements you would want to take a calibration picture the same day along side the rest of the samples you are photographing. Some microscopes go into and out of alignment morning and afternoon, while other scopes are like rocks and you align them once a year it seems. Do you have any feeling for changes in magnifications over time? If I sit down and do a calibration range from magnification settings, can I come in the next week and expect to assume that my electronics are going to be stable enough so I don't have to keep doing these magnification standards on any ongoing basis? Or if I need to do it on an ongoing basis, what might be a ballpark just to keep it in mind... once a month, once a year?

M. Postek: Scanning electron microscopes are used in many, many different applications. So user 1 in the laboratory may be working at high KV with a coated sample, while another user might be working at $1 \mathrm{kV}$ with an uncoated sample. You might have different working distances, different tilts, etc. There are a whole slew of things which may be different. When you 
calibrate the SEM there are certain sequences that you go through. On an AMRAY microscope we typically do it at $12 \mathrm{~mm}$ working distance. I think a Hitachi is $15 \mathrm{~mm}$. To meet those criteria for your general use you must know how the instrument was calibrated. That is why I suggest you talk to the field service engineer and find out what he or she is doing when they do the calibration and how they go about doing it. Are they doing final lens voltage readings to make sure they are at the same working distance, etc? If you did the same thing, that would be the first order of getting good use of your calibration. But if someone comes in and is working at $30 \mathrm{kV}$ and you come in next and you are down at $1 \mathrm{kV}$ you may have lens hysteresis effects you have to be concerned about in that instrument. A simple test to do for yourself is to go into the instrument, put in a sample that is somewhat resilient, and take a high $\mathrm{kV}$ picture. Then go to low $\mathrm{kV}$ and back to high $\mathrm{kV}$ or vice versa and see if the magnifications are the same. If it goes out of focus you have hysteresis effects. Some newer instruments can handle that. They have degaussing capabilities in the software or the hardware. Some do not. The older instruments I am sure do not. You have to be much more careful in many cases than with TEMs. I think the SEMs have been run and built more sloppily in general than the TEMs. In the semiconductor industry these guys are pumping product through the instruments with the same conditions day after day after day at the same working distance, the same accelerating voltage, and so on. Once they get a good calibration of that instrument they can do that and they can be very precise day by day by day. For general laboratory instrumentation you are doing a lot of different things. So you are going to have to check that out for yourself with your own application. If you have a good calibration you will be fine. But if you change working distance, and it's not compensating perfectly, for the long working distance verses the calibration point, you will have lens hysteresis. These kinds of things are going to kill you. You have to be careful about them. Q: I have a question about SEM calibration. Given the physics of the signal generation in the SEM rather than just the operating parameters of the instrument, how much effect does the sample itself have on calibration? How important is this in a service lab where we will have a person doing high $\mathrm{Z}$ materials and the next person is going to be using low- $Z$ biologicals?

M. Postek: In answer to your question, that's part of the 6-hour lecture! (Laughter) Magnification calibration, that's your $\mathrm{x}$ and $\mathrm{y}$ scans and accelerating voltage as long as you have the hysteresis problem dealt with, is not an issue, because you are looking at a pitch. That is the same point on the left side of a line or the center of a line to the same point of another line at a similar location. So you are not dealing with effects of the sample itself when you are doing the calibration. Your magnification calibration, baring these other things I just discussed, should be good. If you are looking at the width measurement of one of your nanotubes for example, you are dealing with additive electron beam effects rather than the effects being compensated for-like they are in a pitch measurement. This is where the physics has to be dealt with. This is where you have to have electron beam modeling to get accurate measurements. You can get lots of precise measurements time after time after time but accurate measurement is something where you are looking for the actual edge. What are you looking at? And you have to physically understand the electron beam effects, the accelerating voltage effects, and these kinds of things relative to the material that you are dealing with. So that is the next step which we didn't get to today.

\section{Endnotes:}

1. Contribution of the National Institute of Standards and Technology. This work was supported (in part) by the National Semiconductor Metrology Program at the National Institute of Standards and Technology; not subject to copyright.

2. Postek, M. T. and Joy, D. C. 1987. Submicrometer Microelectronics Dimensional Metrology: Scanning Electron Microscopy. NBS Journal of Research 92 (3): 205-228.

3. Postek, M. T., Vladar, A. E., Jones, S. N. and Keery, W. J. 1993. Scanning electron microscope magnification calibration interlaboratory study MSA Proceedings 776-777.

4. Postek, M. T., Vladar, A. E., Jones, S. and Keery, W. J. 1993. Report on the NIST Low Accelerating Voltage SEM Magnification Standard Interlaboratory Study. Proceedings SPIE 1926:268-286.

5. Postek, M. T. Vladar, A. E., Jones, S. and Keery, W. J. 1993. Interlaboratory study on the lithographically produced scanning electron microscope magnification standard prototype. NIST J. Res. 98:447-467.

6. Postek, M. T. 1989. Scanning Electron Microscope-based Metrological Electron Microscope System and New Prototype SEM Magnification Standard. Scanning Microscopy 3(4):1087-1099.

7. Postek, M. T., A. E. Vladar and J. Villarrubia. 2000 . Is a production critical scanning electron microscope linewidth standard possible? Proc. SPIE $3988: 42-56$.

\section{Microscopy and Microanalysis}

\section{Table of Contents Preview}

Volume 8, Number 5, October 2002

\section{Biological Review Paper}

- The Pros and Cons of Apoptosis Assays for use in the Study of Cells, Tissues, and Organs, M. Watanabe, M. Hitomi, K. van der Wee, F. Rothenberg, S.A. Fisher, R. Zucker, K.K.H. Svoboda, E.C. Goldsmith, K.M. Heiskanen and A.-L. Nieminen

\section{Materia/s Applications}

-Detection, Distribution, and Quantification of Carbon in Steel Microstructures by PEELS, E.S.K. Menon and A.G. Fox

- Characterization of Magnetic Nanoparticles using Energy-Selected Transmission Electron Microscopy, M.J. Sayagués, T.C. Rojas, A. Fernández, R.E. Dunin-Borkowski, R.C. Doole, and J.L. Hutchinson

-HRTEM Image Simulations for the Study of Ultra-Thin Gate Oxides, S.T. Taylor J. Mardinly, and M.A. O'Keefe

- Measuring Thickness Changes in Thin Films Due to Chemical Reaction by Monitoring the Surface Roughness with In Situ Atomic Force Microscopy, L.Y. Beaulieu, A.D. Rutenberg, and J.R. Dahn

Microanalysis

- Compositional Averaging of Continuum Intensities in Multi-Element Compounds, J.J. Donovan \& N.E. Pingitore, Jr. News and Commentary

- Calendar of Meetings and Courses

- Net Notes

MSA members receive both Microscopy Today and Microscopy and Microanalysis FREE! 